\title{
Studies of Gas-Particle Interactions in a Microgravity Flow Cell
}

\author{
Michel Louge ${ }^{1 a}$, Haitao Xu ${ }^{1 b}$, James Jenkins ${ }^{2}$ and Anthony Reeves ${ }^{3}$ \\ 1 Sibley School of Mechanical and Aerospace Engineering, \\ 2 Department of Theoretical and Applied Mechanics, \\ 3 School of Electrical Engineering, \\ Cornell University, Ithaca, NY 14853
}

\begin{abstract}
We are developing a microgravity flow cell in which to study the interaction of a flowing gas with relatively massive particles that collide with each other and with the moving boundaries of the cell. Unlike Earth-bound flows where the gas velocity must be set to a value large enough to defeat the weight of particles, the duration and quality of microgravity on the Space Station will permit us to achieve suspensions where the agitation of the particles and the gas flow can be controlled independently by adjusting the pressure gradient along the flow and the relative motion of the boundaries. This paper describes the experimental apparatus, outlines a theory and computer simulations to predict the flow, and specifies microgravity requirements for its implementation in Space.
\end{abstract}

\section{INTRODUCTION}

The ability to transport particulate materials predictably and efficiently using a flowing gas is likely to play an important role in the development of Lunar and Martian environments that are hospitable to humans. Lunar soil contains significant amounts of oxygen, chemically bound in various minerals. Through appropriate processing, this oxygen may be recovered for use in propulsion and life support systems. Similarly, it is believed that Martian soil contains significant amounts of water which can be electrolyzed into oxygen and hydrogen, again for propellants and life support. The transport of such granular soils from where they are mined and between stages of their processing is likely to involve pneumatic transport carried out in systems of pipes using flows of the liberated gases.

In that context, we are developing a microgravity flow cell in which to study the interaction of a flowing gas with relatively massive particles that collide with each other and with the moving boundaries of the cell. Unlike Earth-bound flows where the gas velocity must be set to a value large enough to defeat the weight of particles, the duration and quality of microgravity on the Space Station will permit us to achieve suspensions where the agitation of the particles and the gas flow can be controlled independently by adjusting the pressure gradient along the flow and the relative motion of the boundaries.

The facility will permit suspensions to be studied over a range of laminar, steady, fully-developed conditions where viscous forces dominate the gas flow. In particular, forces proportional to the density of the fluid will be nearly eliminated. Within that range, we plan to characterize the viscous dissipation of the energy of the particle fluctuations and observe the development of localized inhomogeneities that are likely to be associated with the onset of clusters. These measurements and observations will contribute to an understanding of the essential physics of pneumatic transport.

Recently, an appreciation has developed for the influence of collisional interactions among particles, both in suspensions where the flow is laminar (Sinclair \& Jackson, 1989) and turbulent (e.g. Louge, Mastorakos \& Jenkins, 1991; Dasgupta, Sundaresan \& Jackson, 1994; Bolio, Yasuna \& Sinclair, 1995; Boëlle, Balzer, and Simonin, 1995). Collisions between such particles can transfer a significant amount of momentum within the flow and at the boundaries. This provides an additional resistance to the passage of the gas, but also introduces a mechanism that promotes more homogeneous flows and, at least in small diameter pipes, may forestall the development of clusters. 
A crucial parameter in such suspensions is the fluctuating kinetic energy or agitation of the particles. It is with this parameter that the solid phase can transmit momentum through an effective viscosity. Sangani, Mo, Tsao and Koch (1996) have recently determined the contribution of the viscous forces of the gas to the dissipation of particle fluctuation energy in random flights of particles between collisions. They do this over a range of concentrations for simple shearing flows in which the particle Reynolds number is small and the Stokes number is greater than one. We have extended their theory to inhomogeneous, threedimensional fully-developed, steady shearing flows in practical wall-bounded devices. This involves the introduction of energy transport due to spatial gradients and the extension of boundary conditions for bumpy frictional walls to systems with large slip velocity at the boundaries.

We begin with a description of the experimental apparatus. We then show preliminary images obtained on the KC-135 microgravity aircraft. We compare computer simulations with theoretical predictions for the mean and fluctuating velocities profiles in flows that we expect to produce in the apparatus. Finally, we outline microgravity requirements for the prescribed experiments.

\section{APPARATUS}

For this project, the Glenn Research Center is modifying an instrument that we have used to investigate mechanisms of granular segregation in microgravity (Louge, et al, 1999). The instrument consists of a flow channel in the shape of a race track bounded by a stationary shell and a moving inner wall driven by a chain (Fig. 1). Cylindrical bumps attached to the moving and stationary boundaries provide the surface texture necessary to produce the desired granular agitation in the interior.

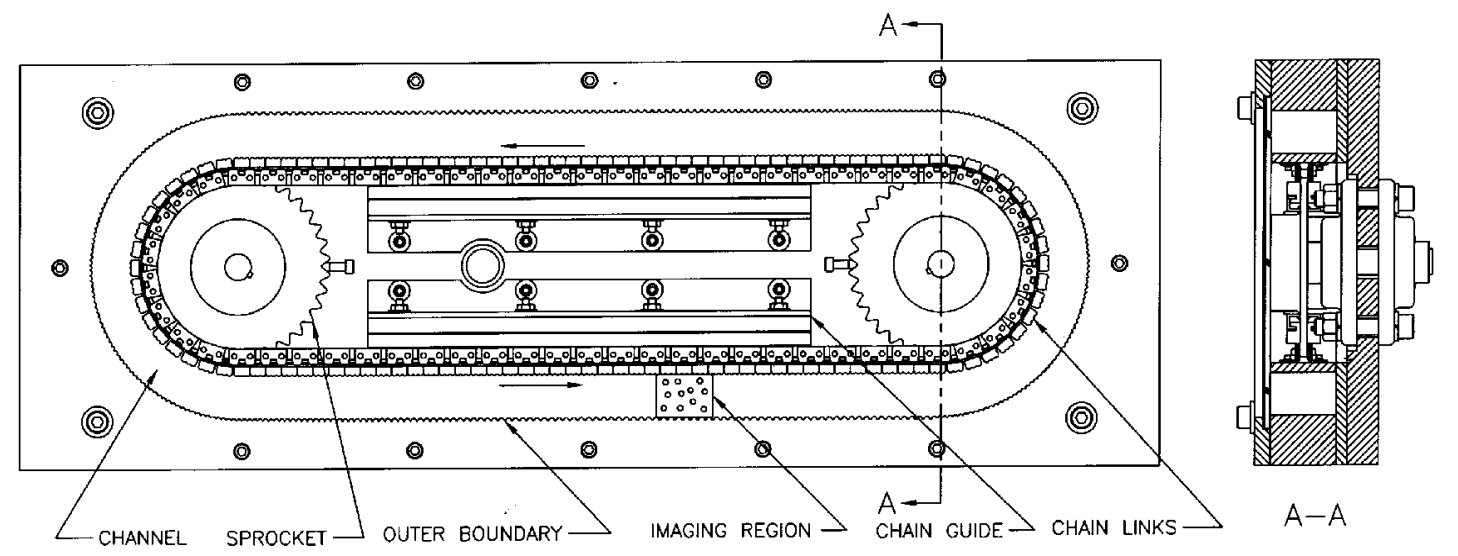

FIGURE 1. Prototype of the Microgravity Shear Cell. The motor and gearbox are omitted for clarity.

In microgravity, this shearing cell will produce a range of flows where solids and gases experience only drag and collisional interactions and where gas flow rates are small enough that gas velocity fluctuations will not be turbulent. On Earth, such an experiment could only be carried out with neutrally buoyant spheres in a liquid. In that case, however, forces proportional to the ratio of fluid to particle densities (lift, added mass, history) would mask the fundamental drag and collisional effects that we intend to isolate.

In the absence of an interstitial fluid, individual impacts are so fast that the only time scale governing the granular phase is the inverse of $\gamma$, the shear rate imposed by the moving boundary. At small particle Reynolds numbers $\operatorname{Re}=\rho \gamma \mathrm{d}^{2} / \mu$, the gas further introduces a viscous relaxation time $\theta=\rho_{\mathrm{S}} \mathrm{d}^{2} / 18 \mu$, where $\rho_{\mathrm{S}}, \mathrm{d}, \rho$ and $\mu$ are, respectively, the material density of the spheres, their diameter and the gas density and viscosity. In simple shear flows, Sangani, Mo, Tsao and Koch (1996) have calculated values of the limiting Stokes number $\mathrm{St}=\gamma \theta$ at which the particle fluctuation energy is equally dissipated by viscous and collisional interactions. Far above this limit, the shear rate is sufficient to ignore viscous drag on the spheres. In contrast, in our experiments we will reduce the boundary speed until the Stokes number becomes small enough for the gas to affect the balance of fluctuation energy of the spheres. However, the Stokes number will not be so small that gas velocity fluctuations dominate particle motion. 
In this apparatus, the simplest flow consists of merely letting the particles entrain the gas. In this case, reductions in the boundary speed gradually produce smaller Stokes numbers and thus greater viscous dissipation of the particle agitation. Because in this simple flow the mean relative velocity between the particles and the gas is small, the fluctuations in gas velocity are correlated with those of the particles. Consequently, measurements of the instantaneous particle velocities are sufficient to determine how interstitial air affects the fluctuating velocities of the spheres.

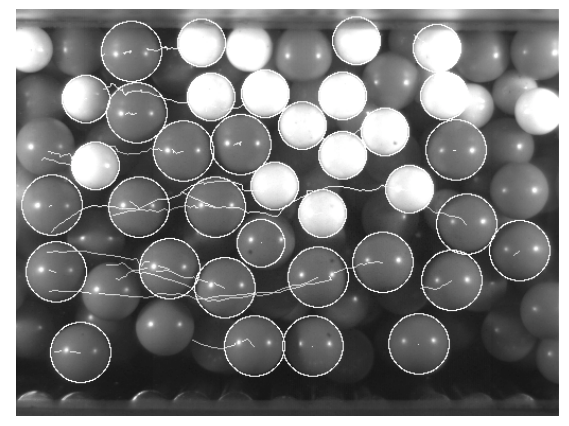

FIGURE 2. A typical image acquired on the $\mathrm{KC}-135$ aircraft with a mixture of $10 \%$ by volume of $3 \mathrm{~mm}$ ceramic spheres and $30 \%$ by volume of $4 \mathrm{~mm}$ acrylic spheres. Circles and lines are superimposed to indicate the location and trajectory of detected spheres. The moving boundary can be discerned at the bottom of the picture.

For these measurements, we will employ an imaging technique that we have demonstrated in similar granular flows on the KC-135 microgravity aircraft (Louge, et al, 1999). There, a high speed video camera was used to observe a flowing binary mixture of grains through lateral windows and particle velocity statistics were extracted using computer tracking (Fig. 2). In Fig. 3, we compare the corresponding profiles of fluctuating velocities with predictions of computer simulations for the entire apparatus.

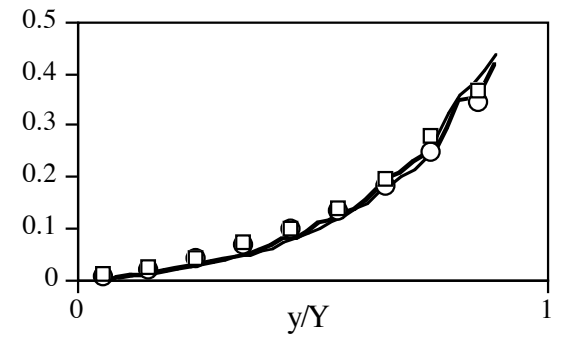

FIGURE 3. Transverse profiles of mean velocity relative to the chain speed $U$ for the conditions of Fig. 2. The abscissa is the distance from the stationary bump centers relative to the separation Y between bump centers of the stationary and moving boundaries. The symbols and lines are experimental measurements and predictions of the simulations, respectively. The circles and thin line are for acrylic spheres; the squares and thick line for ceramic.

Because of the relatively short duration and high levels of microgravity on the KC-135 aircraft, we had to conduct those experiments at boundary speeds high enough for the residual gravity to produce negligible gradients of the normal granular stress. Unfortunately, in that case, granular velocities were too high for the gas to affect the motion of individual grains. Thus, definitive experiments able to reveal the role of the interstitial gas must wait for the availability of long duration and high quality microgravity on the Space Station. For now, as the following section indicates, we employ theory and computer simulations to specify experimental conditions and to design the shearing cell.

\section{THEORY AND SIMULATIONS}

Because the continuum theory of Sangani, Mo, Tsao and Koch (1996) concerns unbounded simple shearing flows of constant agitation, it must be extended in order to apply to bounded flows. To that end, we have developed a new theory for inhomogeneous, three-dimensional, fully-developed, steady shearing flows in practical wall-bounded devices. This involves the introduction of granular fluctuating energy transport due 
to spatial gradients and the extension of boundary conditions for bumpy frictional walls to systems with large slip velocity at the boundaries.

For fully-developed, steady flows, we write the corresponding momentum balances for the solids as

$$
0=\beta(\mathrm{v}-\mathrm{u})-\frac{\partial \mathrm{p}_{\mathrm{s}}}{\partial \mathrm{x}}+\frac{\partial \tau_{\mathrm{xy}}}{\partial \mathrm{y}}+\frac{\partial \tau_{\mathrm{xz}}}{\partial \mathrm{z}}
$$

and for the gas,

$$
0=\beta(\mathrm{u}-\mathrm{v})-\frac{\partial \mathrm{p}_{\mathrm{g}}}{\partial \mathrm{x}}+(1-\mathrm{v}) \mu\left(\frac{\partial^{2} \mathrm{v}}{\partial \mathrm{y}^{2}}+\frac{\partial^{2} \mathrm{v}}{\partial \mathrm{z}^{2}}\right)
$$

where $\mathrm{u}$ and $\mathrm{v}$ are, respectively, the solid and gas velocities in the flow direction $\mathrm{x}, \mathrm{v}$ is the solid volume fraction, $\mathrm{p}_{\mathrm{g}}$ is the gas pressure, and the directions $\mathrm{y}$ and $\mathrm{z}$ are perpendicular to the bumpy boundaries and flat side walls, respectively. The drag coefficient $\beta=18 \mu \nu R_{d}(v) / d^{2}$ accounts for the presence of nearby grains through the term $R_{d}(v)$ (Koch and Sangani, 1999). In these flows, the gas does not perturb the distribution of fluctuating velocities of the grains as long as the Stokes number is much greater than one. In the event, following Jenkins and Richman (1985), the solid pressure is $p_{S}=(1+4 G) v \rho_{S} T$, where $\mathrm{G} \equiv v \mathrm{~g}_{12}(v)$ incorporates the Carnahan and Starling (1979) pair distribution function $\mathrm{g}_{12}(v)=(2-v) / 2(1-v)^{3}$. The scalar $\mathrm{T} \equiv(1 / 3) \overline{u_{i} \mathrm{u}_{\mathrm{i}}}$ is the "granular temperature", a local measure of the fluctuating velocity $u^{\prime}$ i of the flow spheres. The granular stress tensor in Eq. (1) is

$$
\tau_{i j}=\kappa S_{k k} \delta_{i j}+2 \eta \hat{S}_{i j}
$$

where $\kappa=(8 / 3 \sqrt{\pi}) v \rho_{\mathrm{S}} \mathrm{d} \sqrt{\mathrm{T}} \mathrm{G}, \eta=(3 \kappa / 5)\left[1+(\pi / 12)(1+5 / 8 \mathrm{G})^{2}\right], \delta_{\mathrm{ij}}$ is the Kronecker symbol and $\mathrm{S}_{\mathrm{ij}} \equiv(1 / 2)\left(\partial \mathrm{u}_{\mathrm{i}} / \partial \mathrm{x}_{\mathrm{j}}+\partial \mathrm{u}_{\mathrm{j}} / \partial \mathrm{x}_{\mathrm{i}}\right)$ is the rate of strain tensor for the grains with deviatoric part $\hat{\mathrm{S}}_{\mathrm{ij}}$. We then write an equation for the balance of fluctuating kinetic energy of the spheres,

$$
0=-\frac{\partial \mathrm{q}_{\mathrm{y}}}{\partial \mathrm{y}}-\frac{\partial \mathrm{q}_{\mathrm{z}}}{\partial \mathrm{z}}+2 \tau_{\mathrm{xy}} \mathrm{S}_{\mathrm{xy}}+2 \tau_{\mathrm{xz}} \mathrm{S}_{\mathrm{xz}}-\mathrm{D}_{\text {inelastic }}-\mathrm{D}_{\mathrm{viscous}}
$$

where the flux of fluctuation energy is

$$
\mathrm{q}_{\mathrm{i}}=-\frac{3}{2} \kappa\left[1+(9 \pi / 32)(1+5 / 12 \mathrm{G})^{2}\right] \frac{\partial \mathrm{T}}{\partial \mathrm{x}_{\mathrm{i}}} .
$$

The fluctuating kinetic energy is dissipated through inelastic collisions,

$$
D_{\text {inelastic }}=\frac{24}{\sqrt{\pi}}(1-\mathrm{e}) \vee \rho_{\mathrm{s}} \mathrm{T}^{3 / 2} \mathrm{G} / \sigma,
$$

and through viscous interactions with the gas,

$$
D_{\text {viscous }}=54 \mu \nu R_{\text {diss }}(v) / d^{2},
$$

where $\mathrm{R}_{\mathrm{diss}}$ is a function of $v$ capturing effects of nearby spheres, gas velocity fluctuations, and the breakdown of lubrication theory at small grain spacing (Sangani, et al., 1996). In Eq. (6), e is an effective coefficient of restitution incorporating frictional dissipation of fluctuating energy (Zhang, 1993).

At the flat side walls, we employ the boundary conditions derived by Jenkins (1992) for the granular stress and by Jenkins and Louge (1997) for the granular flux of fluctuating kinetic energy, assuming that all grains are slipping. At the bumpy boundaries, we employ the conditions calculated by Richman and Chou (1988) for smooth bumps and account for frictional interactions by adding the results of Jenkins (1992) and Jenkins and Louge (1997). For the gas, we enforce the customary no-slip condition at all boundaries. We then solve the elliptical system of non-linear PDEs (1) through (7) subject to the boundary conditions in a two-dimensional grid (y,z) using the method of Peaceman and Rachford (1955) and Brian (1961). 
In an attempt to corroborate the theory, we add viscous drag to an existing molecular dynamic simulation of the entire cell, where grains are followed individually through successive collisions (Louge, et al, 1999). Because of the relatively large number of spheres in the cell, we do not employ the methods of Brady and Bossis (1988), Ladd (1990) or Mo and Sangani (1994). Instead, we incorporate the gas in a cruder iterative way, see for example, Tsuji, Kawaguchi and Tanaka (1993) or Boëlle, Balzer and Simonin (1995). To distinguish effects of the gas on the mean and fluctuating velocities of the grains, we invoke the coefficients $R_{d}$ for the drag induced by the mean slip velocity and $R_{\text {diss }}$ for the drag involving its fluctuations. In these simulations, the individual grains first reach a steady flow without consideration for the gas. Then, the corresponding velocity and volume fraction profiles are incorporated in Eq. (2), which is solved numerically for the mean gas velocity profile. In turn, the corresponding drag on the spheres affects the granular force balance in the simulations. The iterative process is repeated until convergence.

As Fig. 4 illustrates, the theory and simulations agree well. Further, it is possible to design an experiment where reductions in the mean Stokes number clearly amplify the dissipation of granular agitation, while the interstitial gas is merely present in the cell rather than being forced through the flow.

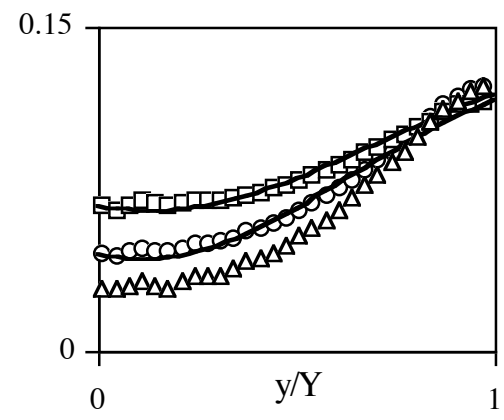

FIGURE 4. Profiles of grain fluctuation velocity relative to the speed of the boundary with $\partial \mathrm{p}_{\mathrm{g}} / \partial \mathrm{x}=0$. The triangles, circles and squares represent $\mathrm{St}=25,50$ and 260, respectively. The symbols and lines represent predictions of simulations and theory, respectively.

\section{MICROGRAVITY REQUIREMENTS}

For these experiments, the theory and simulations warn that the dimensionless microgravity along the flow direction must not exceed the level where it begins to affect granular motion,

$$
\mathrm{g} \mathrm{d} / \mathrm{U}^{2}<510^{-5} \text {. }
$$

Then, in order to produce visually recognizable sphere movement, the typical rectilinear displacement between two consecutive frames should not exceed the sphere radius. Thus, with a maximum grain velocity magnitude of order the speed $\mathrm{U}$ of the moving wall, the video frame rate $\mathrm{f}$ must be at least

$$
\mathrm{f} \geq \mathrm{U} /(\mathrm{d} / 2)
$$

In addition, the required microgravity time is determined by the number $\mathrm{N}_{\mathrm{f}} \approx 10,000$ of video frames necessary to compute accurate grain velocity fluctuation statistics (Louge, et al, 1999), following the establishment of a steady flow. Then, using Eq. (9), the minimum duration of microgravity is

$$
\theta_{\mu \mathrm{g}} \geq \mathrm{N}_{\mathrm{f}}(\mathrm{d} / 2) / \mathrm{U}
$$

TABLE 1. Experimental requirements.

\begin{tabular}{lcccc} 
Stokes number & Boundary speed & frame rate $\geq$ & microgravity $\leq$ & duration $\geq$ \\
\hline 260 & $10 \mathrm{~cm} / \mathrm{sec}$ & $200 \mathrm{~Hz}$ & $520 \mu \mathrm{g}$ & $50 \mathrm{sec}$ \\
100 & $4 \mathrm{~cm} / \mathrm{sec}$ & $80 \mathrm{~Hz}$ & $80 \mu \mathrm{g}$ & $130 \mathrm{sec}$ \\
50 & $2 \mathrm{~cm} / \mathrm{sec}$ & $40 \mathrm{~Hz}$ & $20 \mu \mathrm{g}$ & $260 \mathrm{sec}$ \\
25 & $1 \mathrm{~cm} / \mathrm{sec}$ & $20 \mathrm{~Hz}$ & $5 \mu \mathrm{g}$ & $510 \mathrm{sec}$
\end{tabular}


Table 1 indicates the boundary speed, minimum camera frame rate, maximum levels of sustained microgravity and test duration for each of four separate Stokes numbers to be investigated. Clearly, these experiments must wait for the availability of a platform in Space. 


\section{ACKNOWLEDGMENTS}

This research was sponsored by NASA's Microgravity Science and Applications Division under contracts NCC3-468 and NAG3-2112.

\section{REFERENCES}

Boëlle A., Balzer G., and Simonin O. , "Particle-phase stress tensor with gas influence in simple shear dense suspensions," in Gas-Particle Flows, FED-Vol 228, ASME (1995).

Bolio, E. J., Yasuna, J. A., and Sinclair, J. L., "Dilute, turbulent gas-solid flow in risers with particle-particle interactions," AIChE J. 41, 1375-1388 (1995).

Brady J.F. and Bossis G., "Stokesian dynamics," Ann. Rev. Fluid Mech. 20, 111-157 (1988).

Brian P.T.T., "Finite-difference method of high-order accuracy for the solution of three-dimensional transient heat conduction problems," AIChE J. 7, 367-370 (1961).

Carnahan N.F. and Starling K.E., "Equations of State for Non-Attracting Rigid Spheres," J. Chem. Phys. 51, 635636 (1979).

Dasgupta S., Jackson R., and Sundaresan S., “Turbulent gas-particle flow in vertical risers," AIChE J. 40, 215-228 (1994).

Jenkins J.T., "Boundary conditions for rapid granular flows: flat, frictional walls," J. of Applied Mech. 59, 120127 (1992).

Jenkins J.T. and Louge M., "On the flux of fluctuation energy in a collisional grain flow at a flat, frictional wall," Phys. Fluids 9, 2835-2840 (1997).

Jenkins J.T. and Richman M.W., "Grad's 13-moment system for a dense gas of inelastic spheres," Arch. Rat. Mech. Anal. 87, 355-377 (1985).

Koch D.L. and Sangani A.S., "Particle pressure and marginal stability limits for a homogeneous monodisperse gas fluidized bed: Kinetic theory and numerical simulations," submitted to the J. Fluid Mech. (1999).

Ladd A.J.C., "Hydrodynamic transport coefficients of random dispersion of hard spheres," J. Chem. Phys. 95, 3484-3494 (1990).

Louge M.Y., Jenkins J.T., Reeves A., and Keast S., "Microgravity segregation in collisional granular shearing flows," in Segregation in Granular Flows, edited by A. Rosato, Kluver, Dortrecht, The Netherlands (1999), in press.

Louge M.Y., Mastorakos M., and Jenkins J.T. "The Role of Particle Collisions in Pneumatic Transport," J. of Fluid Mech. 231, 345-359 (1991).

Mo G. and Sangani A., "A method for computing Stokes flow interactions among spherical objects and its application to suspensions of drops and porous particles," Phys Fluids 6, 1637-1652 (1994).

Peaceman D.W. and Rachford H.H., "The numerical solution of parabolic and elliptic differential equations," $J$. SIAM 3, 28-41 (1955).

Richman M.W. and Chou C.S., "Boundary effects on granular shear flows of smooth disks," J. Appl. Mech. Phys. (ZAMP) 39, 885-901 (1988).

Sangani A.S., Mo G., Tsao H.-K., and Koch D.L., "Simple shear flows of dense gas-solid suspensions at finite Stokes number," J. Fluid Mech. 313, 309-341 (1996).

Sinclair, J. L. and Jackson, R., "Gas-particle flow in a vertical pipe with particle-particle interactions," AIChE J. 39, 1473-1486 (1989).

Tsao H.-K. and Koch D.L., "Simple Shear flows of dilute gas-solid suspensions," J. Fluid Mech. 296, 211-245 (1995).

Tsuji Y., Kawaguchi T., and Tanaka T., "Discrete particle simulation of two-dimensional fluidized bed," Powder Tech. 77, 79-87 (1993).

Zhang C., "Kinetic theory for rapid granular flows," Ph.D. Thesis, Cornell University (1993). 